\title{
Water Quality Online Monitoring System Integration of Three Gorges Drawdown Areas
}

\author{
Ye Yuan ${ }^{1, \text { a }}$, Mingsheng Shang ${ }^{1, \mathrm{~b}}$, Guoying Wang ${ }^{1, \mathrm{c}}$, Xuerui Zhang ${ }^{1, \mathrm{~d}}$ \\ ${ }^{1}$ Chongqing Institute of Green and Intelligent Technology, Chinese Academy of Sciences, \\ 400714, China \\ ayuanye@cigit.ac.cn, ${ }^{b}$ msshang@cigit.ac.cn, cwangguoyin@cigit.ac.cn, ${ }^{\mathrm{a}} \mathrm{zxr} @$ cigit.ac.cn
}

Keywords: water quality, online monitoring, Three Gorges drawdown.

\begin{abstract}
According to the water quality monitoring need of Three Gorges Drawdown Areas on automation, systematization, we design the overall framework and develop the water quality online monitoring software [1] for water quality online monitoring system based on the Internet of things (TOT) and ArgGIS. The online monitoring is divided into three parts: data perception layer, network data transmission layer, data application service layer. This paper researches how to integrate the water quality automatic monitoring equipment to achieve water data automatic collection, designs the function of the water quality online monitoring software, predicts the water quality with the big data prediction algorithm. The results show that the system is effective.
\end{abstract}

\section{Introduction}

At present, the monitoring method of Three Gorges drawdown areas is traditional, such as analyzed after manual sampling by people. This method not only wastes a lot of manpower and material resources, but also has a low efficiency of data transmission and processing. It can not solve the environmental incidents happened suddenly. And people needs the monitoring is more efficient and more accurate because of the importance and complexity of the Three Gorges Project. Limited to the current level of construction, the traditional monitoring facilities and monitoring methods can not process and analysis the data quickly to reach the goal of rapid response. So we need to introduce and study new monitoring technology and method to build a complete online monitoring system [2]. We can use this system to real-time monitor the water quality continuously. In this paper, we integrate a set of automatic water quality monitoring system based on the Internet of things, and introduce the overall framework of system and the functions of the online monitoring software in detail.

\section{Overall framework of system}

Water quality monitoring system collects the data from the sensor that is placed under the water and then transfers the data to the online monitoring software for storing, processing, analyzing, and displaying though $2 \mathrm{G}$ or $3 \mathrm{G}$ wireless network. We also can observe the whole Three Gorges from the ArgGIS. The system is divided into data perception layer, network data transmission layer and data application service layer. The overall framework is shown in figure 1. 


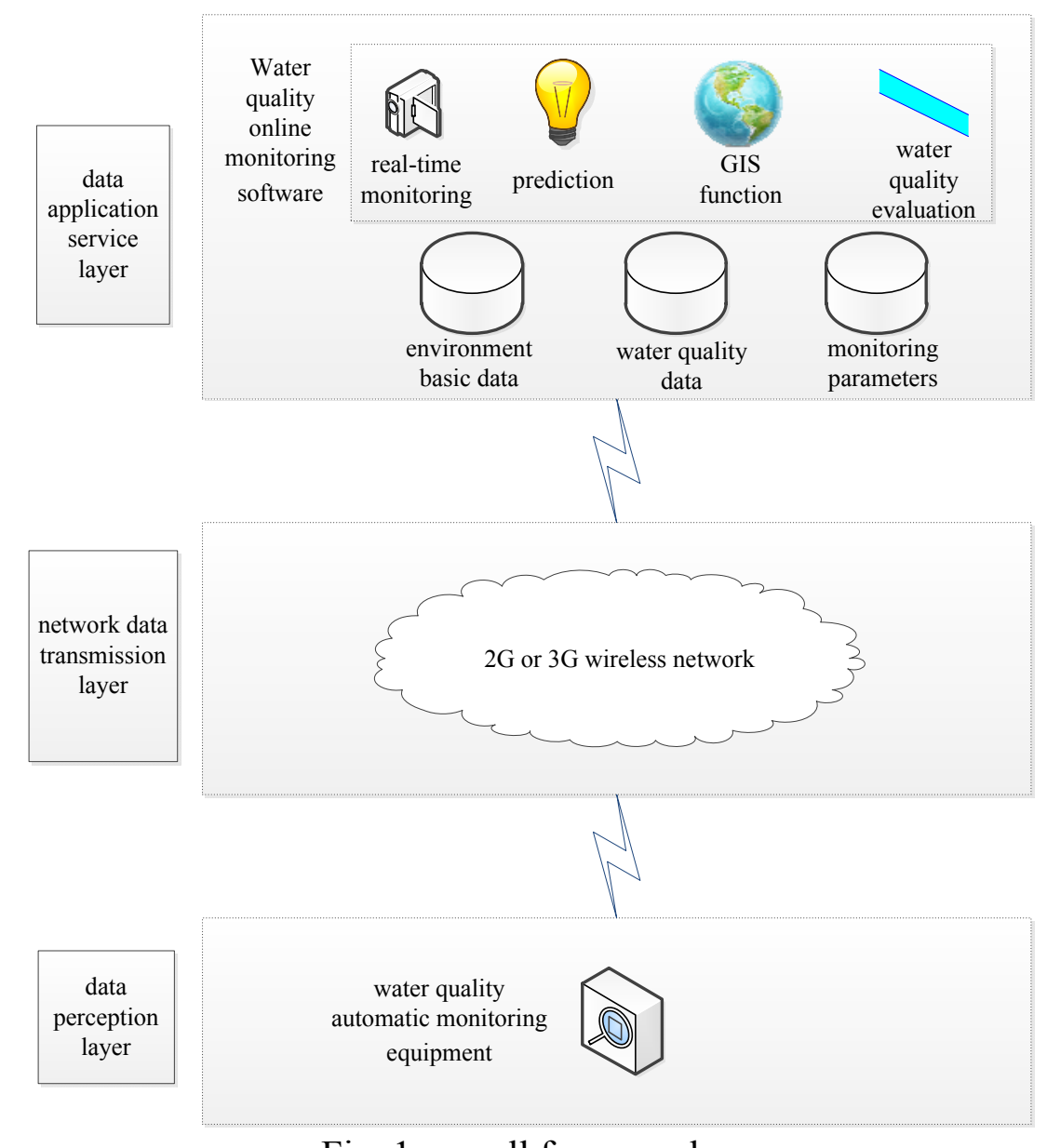

Fig. 1 overall framework

Data perception layer: Installing the water quality monitoring equipment in the Three Gorges drawdown areas for collecting $\mathrm{pH}$, turbidity, dissolved oxygen, electrical conductivity etc. It can provide the basic data of the water quality.

Network data transmission layer: mainly responsible for data transmission from senor to the data application layer. Due to the real-time and safety, we select GPRS as the transmission mode [3].

Data application service layer: responsible for the water data storage, processed, statistical, analysis, graphics displayed, online queried and shared [4].

\section{Hardware integration of automatic monitoring equipment}

In order to adapt the harsh environment of the Three Gorges drawdown areas [5], water quality automatic monitoring equipment must have the characteristics of low power consumption, self-cleaning, solar power supply, storage capacity and so on. The equipment consists of multi parameter water quality sensor [6] which is name as AP7000 developed by Aquaread, data collector name as CR200X, wireless data transmission module name as MD-609G and solar power supply system.

a. AP7000 water quality sensor is shown in Figure 2. It is including water temperature probe, $\mathrm{pH}$ probe, turbidity probe, dissolved oxygen probe, electrical conductivity probe to collect the water environment parameters. The biggest advantage of AP7000 is that a double brush cleaning system is installed to clean all the probes and avoid the probes covered by the algae and water impurities. 

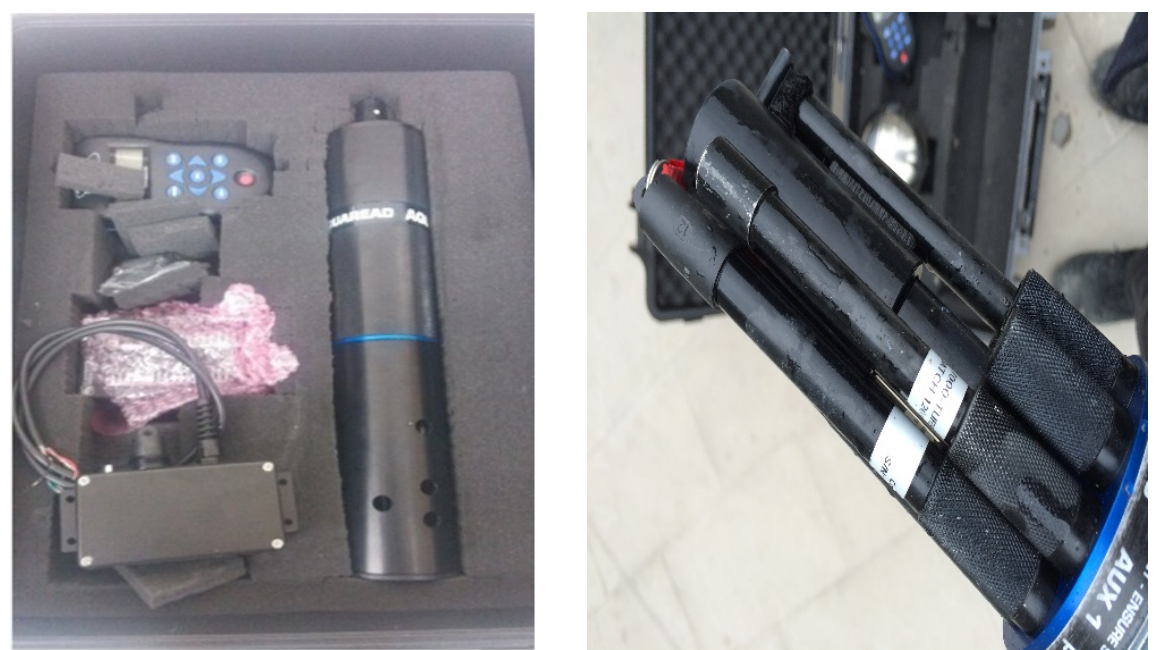

Fig. 2 AP7000

b. CR2000X is the lowest cost and smallest data collector produced by Campbell Scientific company. Single input channel is the best for a sensor and it can save 12800 data at a time of a second scanning frequency. It includes a built-in calibration and can be programmed to adjust the frequency and other parameters of the collector. The details are shown in figure 3.

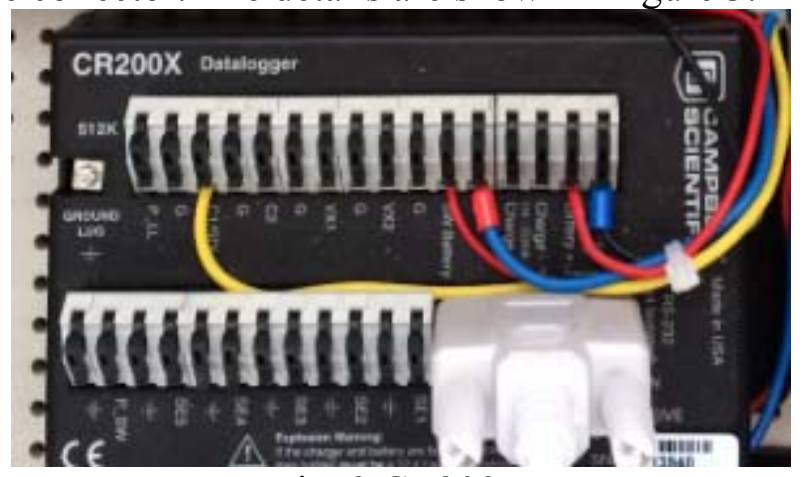

Fig. 3 CR200X

c. MD-609G can transfer data information to a host through wireless network which is connected to the module. It can be widely used in water quality monitoring, meteorological information collection, environmental data collection industry. This module is compatible with a variety of data center software, and low power consumption makes it suitable for mobile portable data transmission and field data monitoring.

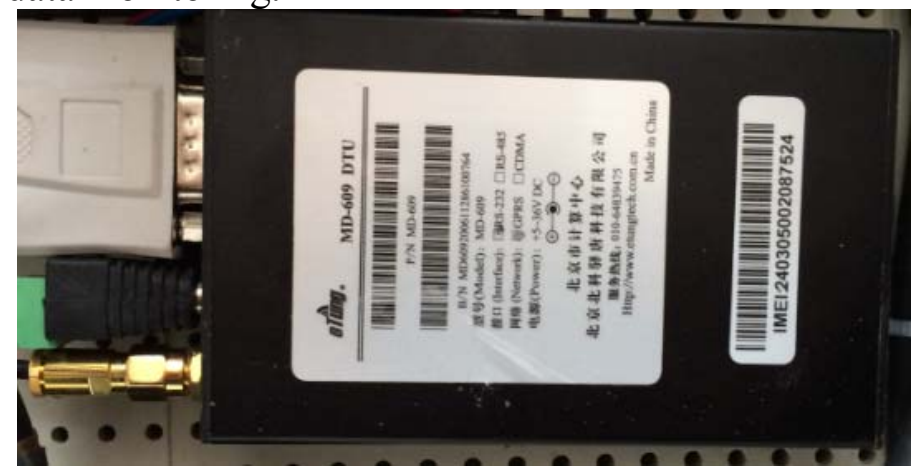

Fig. 4 MD-609G

d. The biggest difficulty of field monitoring is power supply, but solar power supply system can solve this problem. It consists of solar panels, battery and power controllers. The working voltage of AP7000 is $12 \mathrm{~V}$, so the output voltage $12 \mathrm{~V}$ of the battery is chosen for power supply. The battery is charged by the power supply controller and can continuously support the monitoring equipment working normally for a week. The power supply system is shown in figure 5 . 


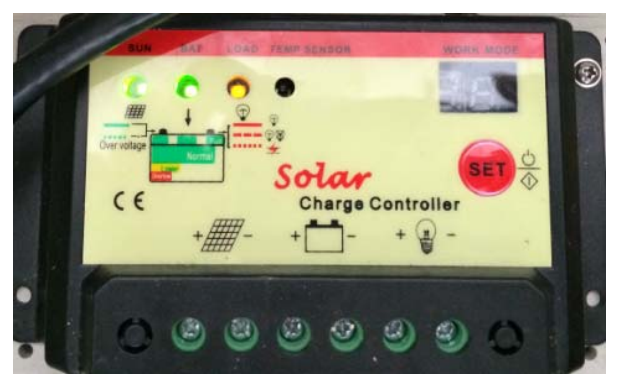

Fig. 5(a) power controllers

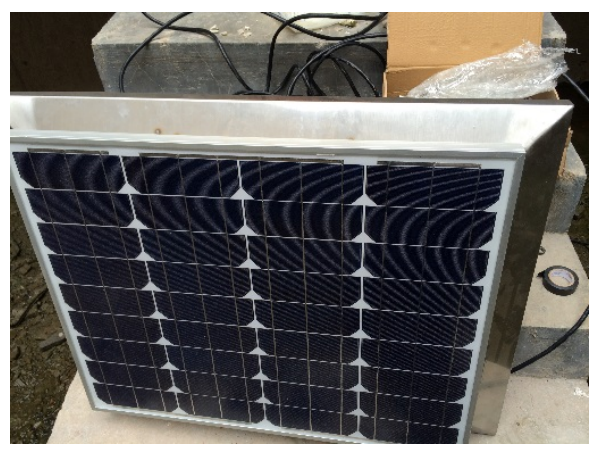

Fig.5(b) solar panels

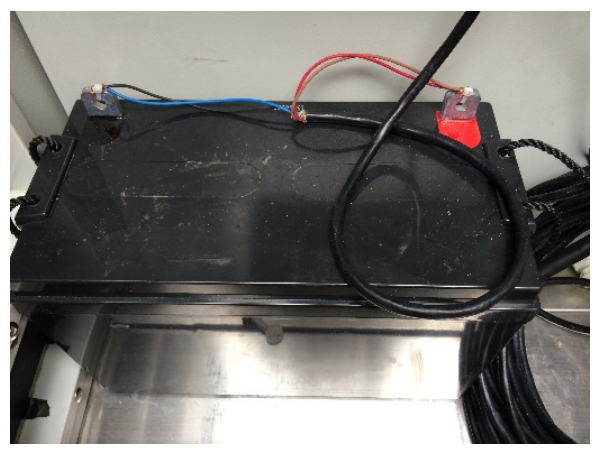

Fig.5(c) battery

\section{Water quality online monitoring software}

This software is mainly responsible for the water quality data storage and provides real-time monitoring results to the administrative department. The software is build by .NET application framework, database is developed by Sql Sever2008 and the GIS function is based on the ArcGIS. The interface of software is shown in figure 6.

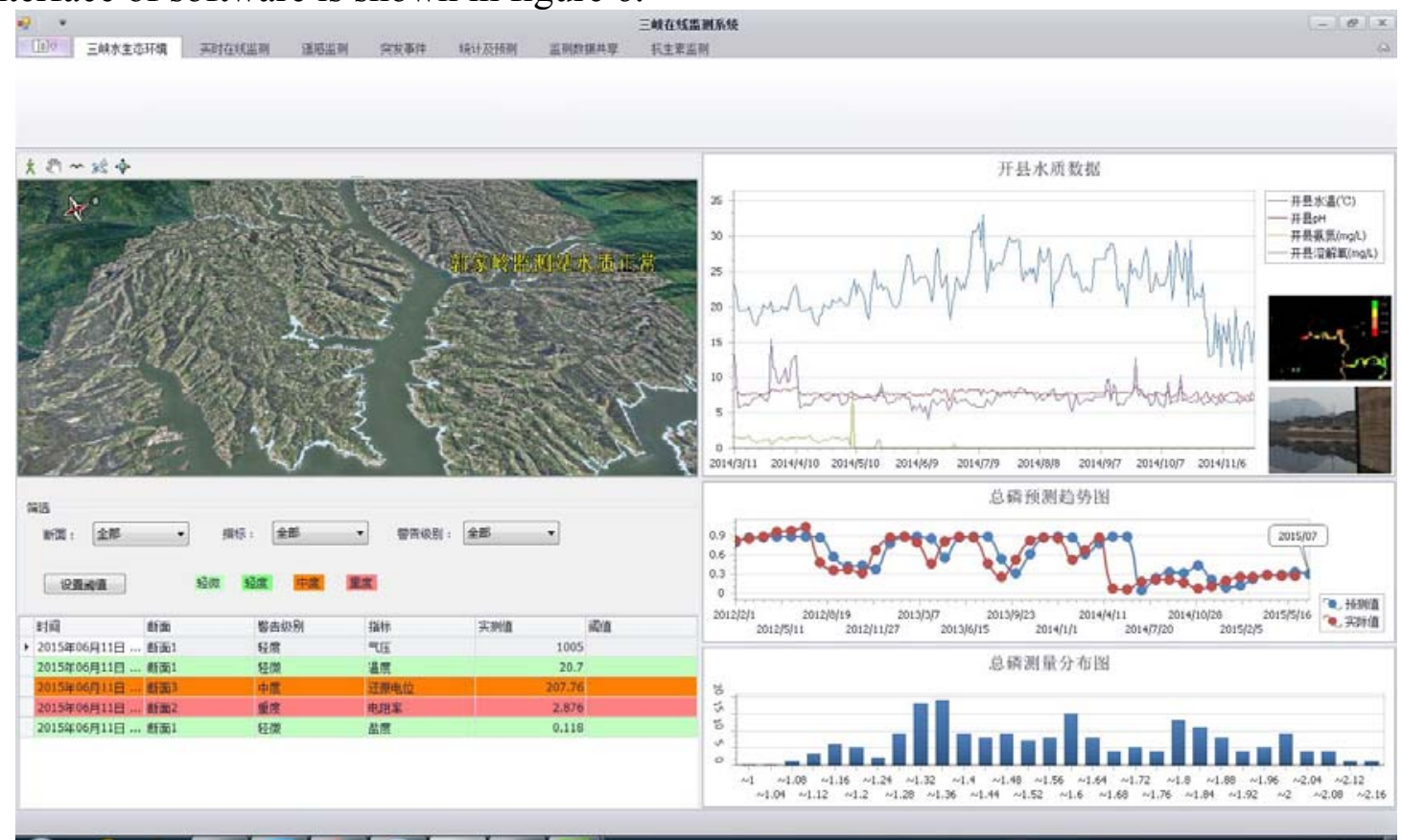

Fig. 6(a) main interface 


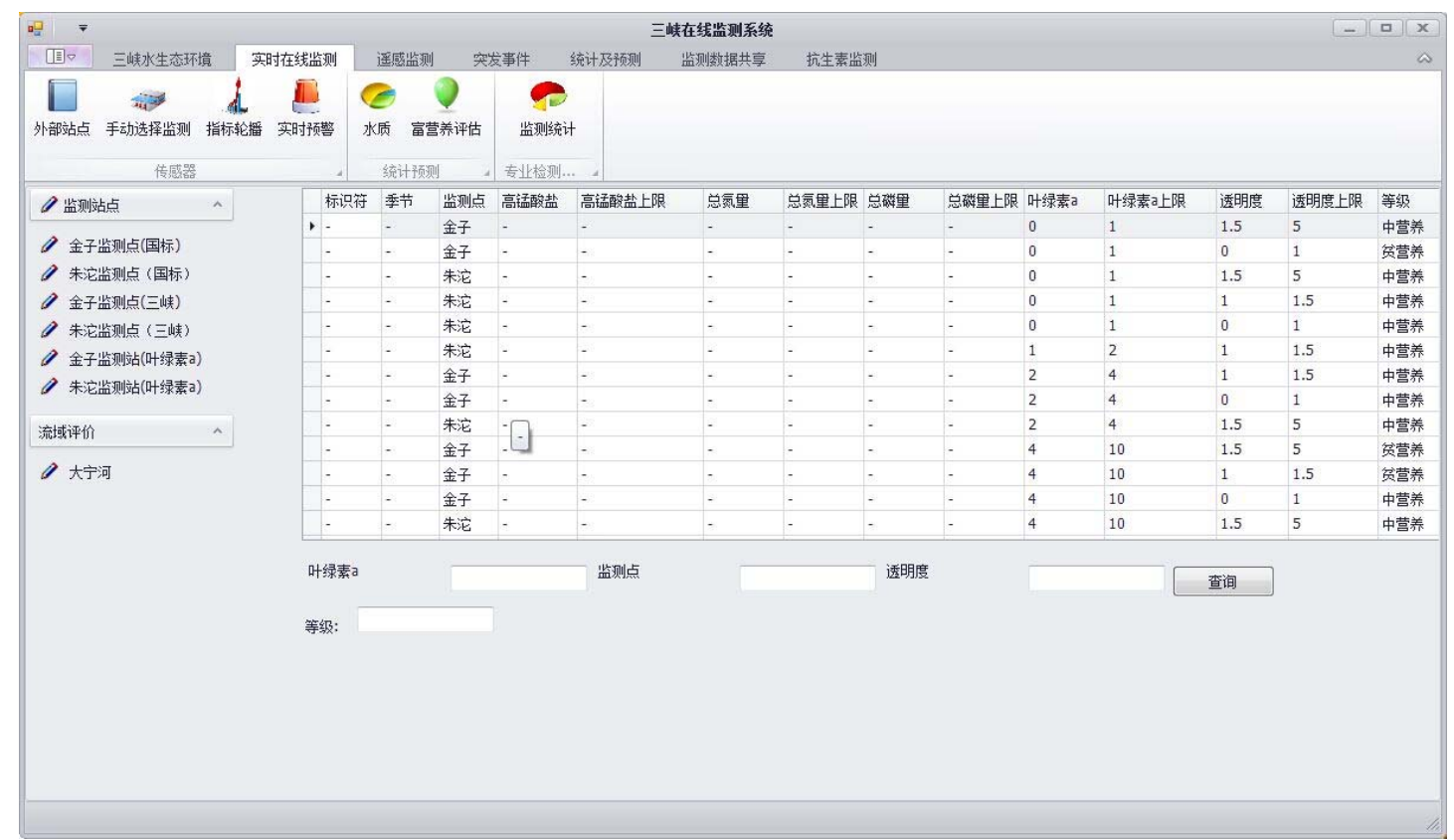

Fig. 6(b) real-time online monitoring interface

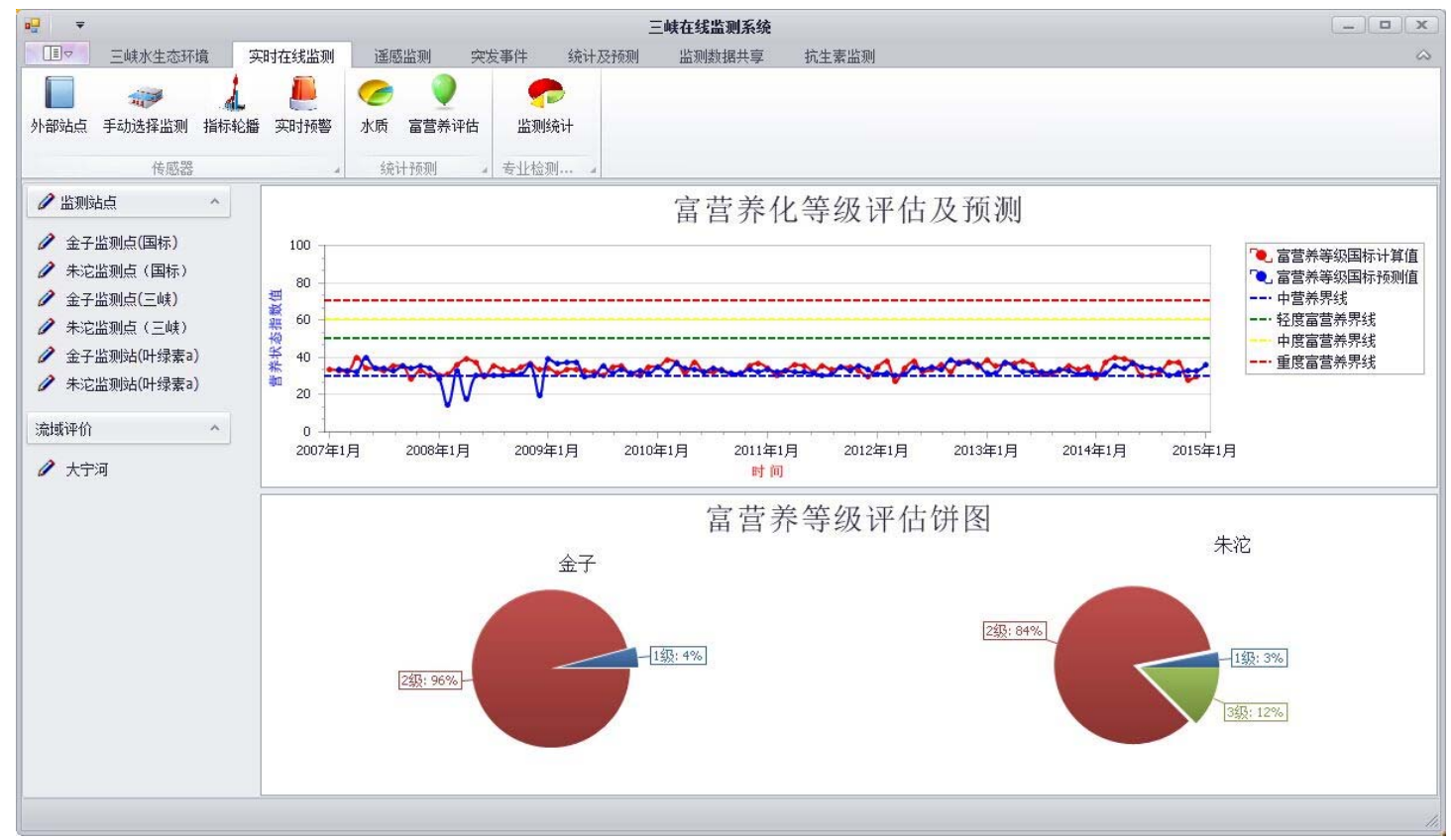

Fig. 6(c) water quality evaluation interface

Water quality online monitoring software includes five main functions: storage management, real-time monitoring, prediction, GIS function, water quality evaluation.

Storage management: Responsible for collecting data, storing data, preprocessing data from monitoring equipment. Water quality data, environment basic data and monitoring parameters can be management in this modular.

Real-time monitoring: Display the water quality data on the interface in real-time, update the sampled data every ten minutes to provide uninterrupted water quality information throughout the day.

Prediction: According to the water quality data, it can predict water quality change over time by using a method combined with periodic water quality and then provide alarm to the management when the water quality index exceeds the threshold [7].

GIS function: Based on the development of ArcGIS, we can observe the whole Three Gorges by using $3 \mathrm{D}$ height data. It also can display the geographic location of the monitoring point. You can 
move, zoom in and out the GIS map to achieve the correspondence between the spatial position and water quality data.

Water quality evaluation [8]: According to the evaluation objective, select the water quality parameters, standards and evaluation methods to evaluate the water quality and then mark the relevant indicators.

\section{Equipment working}

The water quality online monitoring equipment is installed at the Han Feng Lake in Kaixian, a Chongqing County and it is integrated and installed successfully after seven days. The installation diagram is as follows.
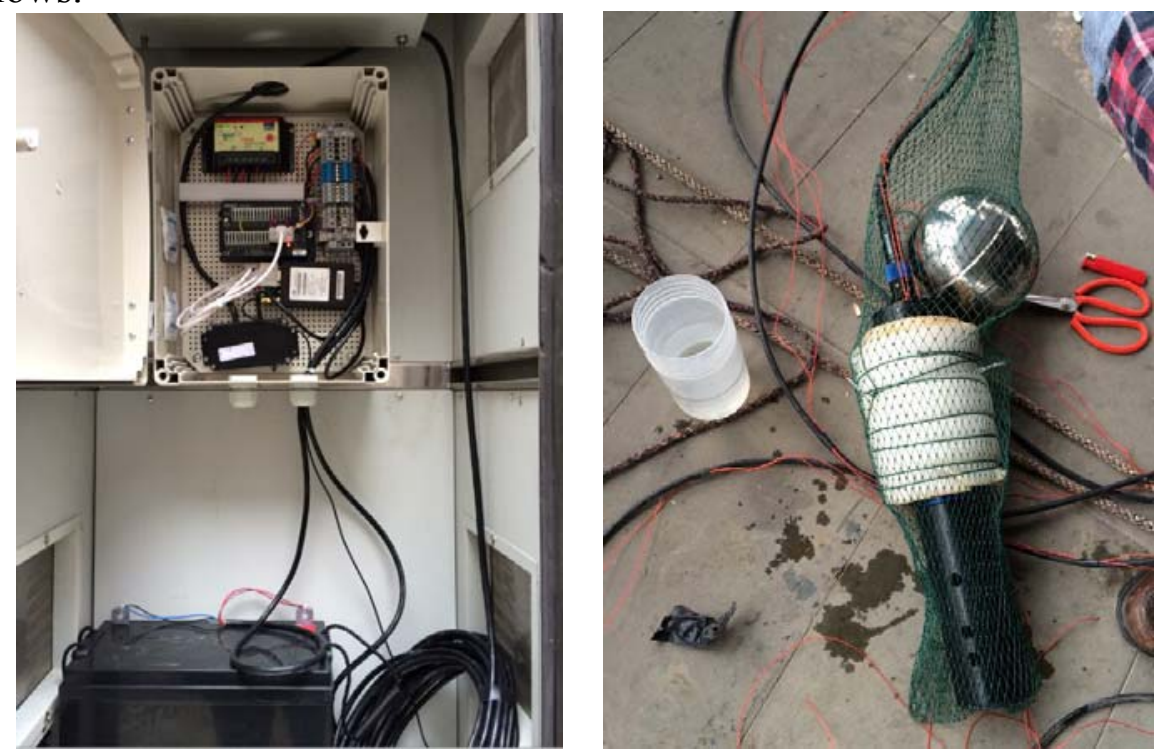

Fig. 7 installation

The solar power supply voltage will fluctuate within a certain range because the intensity of sun is different but we found the voltage is around the $12 \mathrm{~V}$ within normal range. The following figure shows the voltage.

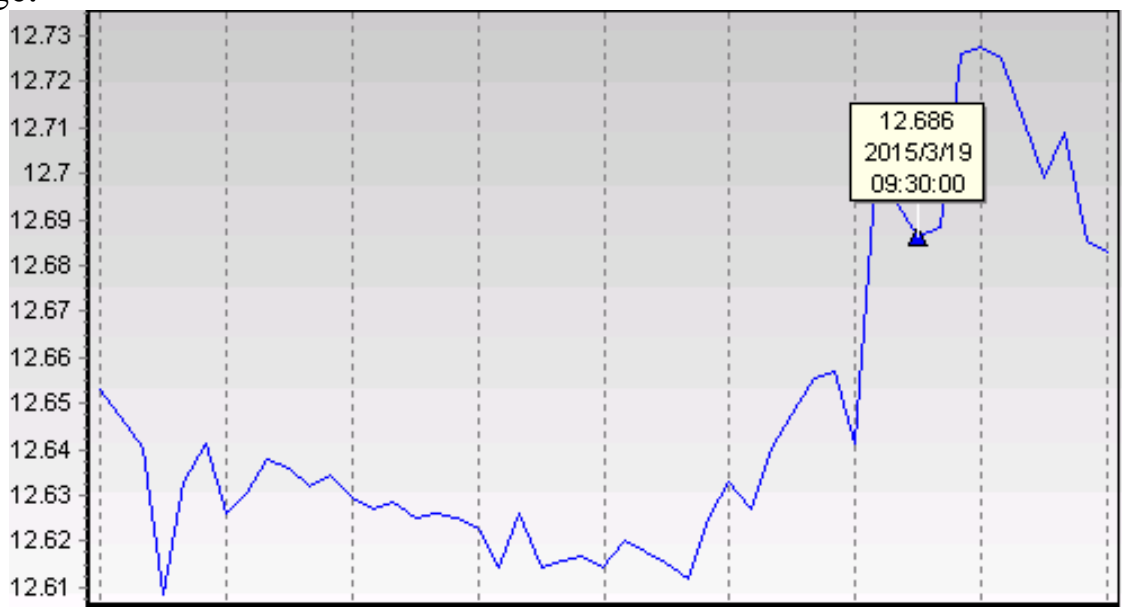

Fig. 8 voltage fluctuation

We set the sampling frequency is 10 minutes [9]. From figure 9, we can see the online monitoring equipment is running well and can monitor and transmit the data according to the requirement. 


\begin{tabular}{|l|l|l|l|l|l|l|}
\hline TIMESTAMP & Temp_C & pH & Cond25_uS & DO_mg & TURB_NTU & NH4_mg \\
\hline 2014-04-01 10:10:00.000 & 18.7 & 7.01 & 1144 & 15.35 & 5.55 & 0.94 \\
\hline 2014-04-01 10:20:00.000 & 18.7 & 7.04 & 1142 & 8.89 & 5.13 & 0.95 \\
\hline 2014-04-01 10:30:00.000 & 18.7 & 7.04 & 1142 & 18.76 & 5.4 & 0.96 \\
\hline 2014-04-01 10:40:00.000 & 18.8 & 7.97 & 1141 & 11.66 & 2.46 & 0.98 \\
\hline 2014-04-01 10:50:00.000 & 18.9 & 7.3 & 1142 & 16.43 & 6.75 & 0.97 \\
\hline 2014-04-01 11:00:00.000 & 19.1 & 7.2 & 1144 & 11.91 & 4.41 & 0.99 \\
\hline 2014-04-01 11:10:00.000 & 19.1 & 7.23 & 1145 & 19.1 & 5.5 & 0.98 \\
\hline 2014-04-01 11:20:00.000 & 19.2 & 7.19 & 1136 & 13.69 & 4.5 & 0.99 \\
\hline 2014-04-01 11:30:00.000 & 19.2 & 7.18 & 1138 & 10.29 & 6.94 & 0.98 \\
\hline 2014-04-01 11:40:00.000 & 19.3 & 7.01 & 1141 & 16.24 & 3.13 & 1.01 \\
\hline 2014-04-01 11:50:00.000 & 19.5 & 7.16 & 1143 & 12.83 & 5.82 & 1.01 \\
\hline 2014-04-01 12:00:00.000 & 19.5 & 7.16 & 1139 & 32.59 & 4.69 & 1.02 \\
\hline 2014-04-01 12:10:00.000 & 19.6 & 7.2 & 1137 & 14.45 & 5.53 & 1.01 \\
\hline 2014-04-01 12:20:00.000 & 19.8 & 7.39 & 1139 & 9.38 & 7.7 & 1 \\
\hline
\end{tabular}

Fig. 9 water quality data

\section{Summary}

According to the water quality monitoring need of Three Gorges Drawdown Areas on automation, systematization, we integrate the water quality automatic monitoring system which has the advantages of real-time monitoring, alarm, spatial visualization etc. The system can monitor the Three Gorges continuously to achieve the goal of real-time monitoring. It can provide the data and support to the management. The system is running well now and the technology has reached the advanced level.

\section{Acknowledgment}

This work is supported by National major science and technology Project (No. 2014ZX07104-006-06), STS Project of Chinese Academy of Sciences (No. KFJ-EW-STS-011).

\section{References}

[1]. Zhao Qing, Wang Li, Yu luji. Water environment quality evaluation index system for the town regions. Proceedings of Second National Academic Conference on Agricultural Environment Science, 2007:544-548.

[2]. Sun Hailin, Li Jufeng, Zhu Yuanyuan. Development and prospect of water quality on-line monitoring system in China [J]. China Environmental Protection Industry, 2009(2):12-16.

[3]. Cui Man, Xue Huifeng, Bu fanbiao, et. Study on monitoring system based on internet of things and cloud computing [J]. Journal of Xi'an Technological University, 2013, 33(7):577-582.

[4]. Wang Jing, Liu Tingtng. Application of wireless sensor network in Tangtze River basin water environment monitoring. Control and Decision Conference(CCDC), 2015 27th Chinese, 5981-5985.

[5]. Yi Yun, Zou Zhihong. An improved synthetic evaluation method on water quality evaluation in city sections of the Three Gorges reservoir area, Grey Systems and Intelligent Services, 2007 IEEE International Conference, 289-293.

[6]. Anvari A, Reyes J.D, Esmaeilzadeh E, Jarvandi A. Designing an automated water quality monitoring system for West and Rhode Rivers. Systems and Information Engineering Design Symposium, 2009 131-136. 
[7]. Nasser N, Ali A, Karim L. An efficient Wireless Sensor Network-based water quality monitoring system. Computer Systems and Applications(AICCSA), 2013 ACS International Conference, $1-4$.

[8]. Wei Chen. Research on water environment automatic monitoring evaluation system for ecological compensation. Remote Sensing, Environment and Transportation Engineering(RSETE), 2011 international Conference, 6576-6579.

[9]. Hu Chengyu, Tian Dijun, Yan Xuesong. Research on placement of water quality sensor in water distribution on systems. Intelligent Control and Automation(WCICA), $201411^{\text {th }}$ World Congress, 3584-3587. 